\title{
ANÁLISE COMPARATIVA ENTRE MÉTODOS DE CONTROLE APLICADOS PARA ATENUAÇÃO DE VIBRAÇÕES EM UMA SUSPENSÃO AUTOMOTIVA
}

\section{COMPARATIVE ANALYSIS OF CONTROL METHODS APPLIED FOR ATTENUATING VIBRATION IN AN AUTOMOTIVE SUSPENSION}

\author{
Luiz Gustavo Pereira Roéfero ${ }^{1}$, Fábio Roberto Chavarette ${ }^{1}$, Thiago \\ Carreta Moro ${ }^{1}$, Roberto Outa ${ }^{2}$ \\ ${ }^{1}$ Universidade Estadual Paulista - UNESP. ${ }^{2}$ Faculdade De Tecnologia De Araçatuba- \\ FATEC \\ E-mail: Igpr98gu@gmail.com
}

RESUMO - A suspensão automotiva é um dos equipamentos presentes em um veículo, sendo usada para preservar a integridade estrutural do mesmo. Quando utilizado, esse dispositivo usa elementos passivos de atuação (normalmente molas e amortecedores) para realizar a atenuação da vibração, entretanto algumas empresas, entre elas a Audi, vem utilizando um controle semiativo para a mesma função alegando que há ganhos com relação aos sistemas convencionais. Outro método normalmente utilizado em suspensões automotivas são os controles ativos, já aplicados na Fórmula 1 pela Willians em 1992. Este trabalho se dedica ao estudo e avaliação da diferença de comportamento de uma suspensão automotiva quando submetida a cada um dos três métodos de controle.

Palavras-chave: Suspensão Automotiva; Controle Ativo; Amortecedor Magneto-Reológico.

ABSTRACT - Automotive suspension is one of the equipment present in a vehicle and is used to preserve its structural integrity. When used, this device uses passive actuation elements (usually springs and dampers) to perform vibration attenuation, however some companies, including Audi, have been using a semi-active control for the same function claiming that there are gains over conventional systems. . Another method commonly used in automotive suspensions is active controls, already applied to Formula 1 by Willians in 1992. This work is dedicated to the study and evaluation of the difference in behavior of an automotive suspension when subjected to each of the three control methods.

Keywords: Automotive Suspension; Active Control; MagnetoRheological damper. 


\section{INTRODUÇÃO}

As suspensões automobilísticas são equipamentos que buscam promover um certo isolamento entre a estrutura do automóvel e as variações de amplitude do trajeto percorrido. De acordo com Picado (1998), sem este equipamento todas as vibrações decorrentes da excitação do pneu do veículo seriam transferidas integralmente ao mesmo, acarretando danos à estrutura e podendo até gerar falhas indesejáveis.

Outro motivo primordial para o uso de suspensões automobilísticas é o conforto dos passageiros do mesmo, segundo AlvarezSánchez (2013), pois vibrações além de não agradarem aos compradores de um veículo automotivo, podem gerar sérias lesões a longo prazo nos ocupantes do automóvel.

Grande parte das suspensões automotivas atuais são compostas de elementos passivos de atuação, normalmente molas e amortecedores acoplados de modo a dissipar a energia proveniente das alterações no solo. Contudo, segundo Cunha e Chavarette (2013), existem outros métodos de controle que permitem a estabilização de uma suspensão automotiva e se caracterizam como métodos ativos e semiativos.

Os sistemas de controle, que são do tipo passivo, simplesmente são responsáveis por absorver parte da energia de vibração, enquanto que, os sistemas de controle ativo, possuem a habilidade de se adaptar a diferentes condições de carregamento e controle dos modos de vibrações de uma estrutura, minimizando assim os efeitos da vibração sobre a mesma, de acordo com Roéfero e Chavarette (2018).

\subsection{Controle Ativo}

A categoria de controle ativo é subdivida em duas: o controle de feedforward (controle de realimentação) e o controle por feedback (controle de alimentação direta), segundo Bueno (2007).

Bueno também explica que o primeiro tipo de método de controle utiliza um sinal de referência para o controlador e um erro de saída, de modo que ajustando o sinal de controle busca-se a diminuição deste erro já citado. Algumas técnicas de controle ativo de ruído (Active Noise Control) utilizam esta estratégia por meio de filtros.

\subsection{Controle Passivo}

Em meio ao grande número de técnicas empregadas para o controle de vibração em sistemas mecânicos, as técnicas de controle passivo têm sido utilizadas em muitos sistemas industriais graças às inúmeras vantagens quando comparadas com as técnicas de controle ativo, como por exemplo, baixo custo de implementação, fácil manutenção e aplicação (DE LIMA, 2007).

Além disso, tais métodos não necessitam de nenhuma alimentação energética externa, fato que garante inerente estabilidade ao sistema e as tornam mais bem adaptadas a aplicações em sistemas industriais de grande porte.

\subsection{Controle Semiativo}

Segundo Tusset et al. (2012), este tipo de controle tem sido estudado recentemente por pesquisadores devido à sua capacidade de combinar vantagens dos controles ativos e passivo. Ele possui a adaptabilidade dos dispositivos de controle ativo sem demandar grandes fontes de energia.

Uma das características dos controles semiativos é de não adicionar, diretamente, energia à estrutura, bem como ter propriedades que variam dinamicamente. De acordo com Roéfero e Chavarette (2018), eles podem ser vistos como dispositivos controláveis porque, apesar de suas propriedades de amortecimento e/ou rigidez serem modificadas, os sistemas semiativos agem passivamente na estrutura.

\section{MATERIAIS E MÉTODOS}

Neste trabalho utilizou-se dois métodos de controle para realizar a atenuação de vibração de uma suspensão 
automotiva. Primeiramente usou-se o Controlador Linear Quadrático (LQR) e, posteriormente, utilizou-se a modelagem numérica de um Amortecedor MagnetoReológico proposta por Bouc-Wen e mostrada por Dyke et al. (1996).
2.1. Modelo Estudado

O modelo estudado neste trabalho é a representação de $1 / 4$ de veículo proposta por Alvarez-Sánchez (2013), sendo mostrada na Figura 1.

Figura 1. Uma figura normal.

Fonte: (LAM; LIAO, 2003).

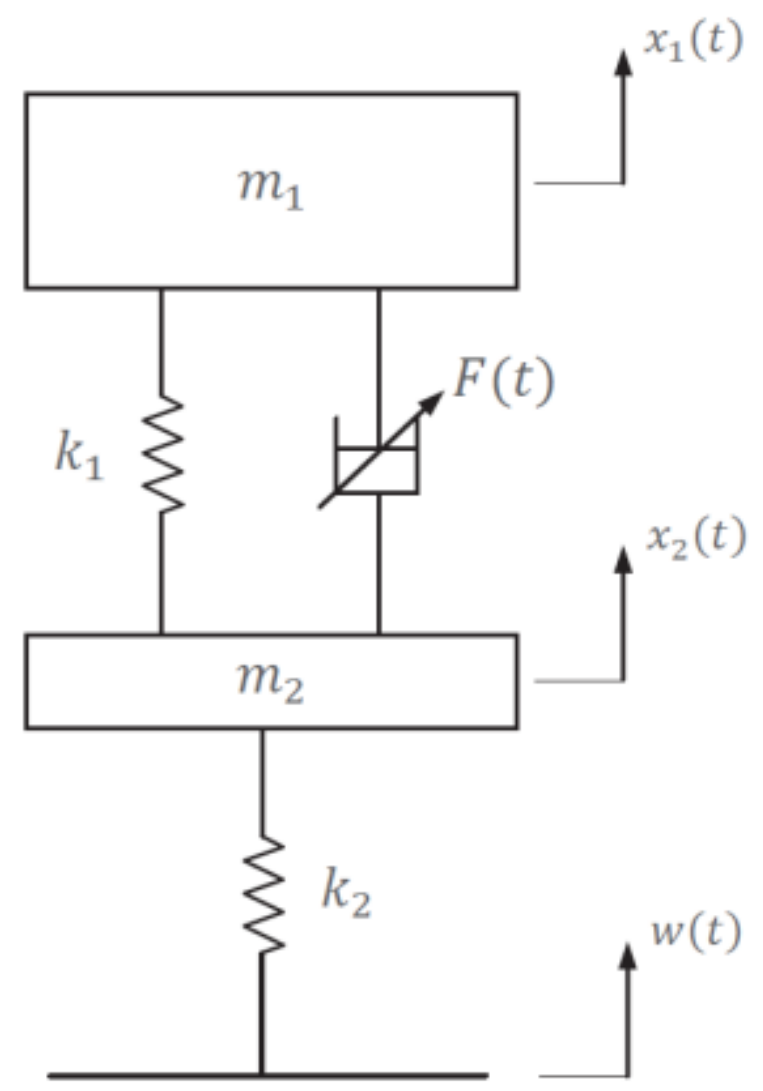

Sendo a evolução temporal descrita pelo A abordagem LQR para obter uma seguinte conjunto de equações proposta por Alvarez-Sánchez (2013): solução ótima do problema de controle tem o seguinte procedimento, segundo Cabral e Chavarette (2015):

$\dot{x_{1}}=y_{1}$

$\dot{y_{1}}=\frac{-k_{1}\left(x_{1}-x_{2}\right)-F}{m_{1}}$

- Represente o modelo na forma de

$\dot{x_{2}}=y_{2}$

$\dot{y}_{2}=\frac{k_{1}\left(x_{1}-x_{2}\right)+F-k_{2}\left(x_{2}-w\right)}{\mathrm{m}_{2}}$ espaço de estado e reescreva-o da seguinte maneira:

$$
\begin{aligned}
& \dot{x}=A x+B u \\
& y=C x
\end{aligned}
$$

Em que a força $F(t)$ é a força desempenhada Sendo, $A \in \Re^{n x n}$ é a matriz pelo amortecedor magneto reológico, quanddinâmica, $B \in \mathfrak{R}^{n x m}$ é a matriz de entrada, utilizado. $C \in \mathfrak{R}^{s x n}$ é a matriz de saída, $x \in \mathfrak{R}^{n}$ é o vetor de estado, $u \in \mathfrak{R}^{m}$ é o lei de controle, $y \in \mathfrak{R}^{s}$ é o vetor de saída. 
- Defina as condições iniciais $x(0)=x_{0}$ e escolha os coeficientes das matrizes de ponderação definidas positivas $Q$ e $R$, que determinam a importância relativa do estado $x(t) \mathrm{e}$ o vetor de controle $u(t)$, respectivamente.

- Resolva a equação de Riccati dada por:

$A^{T} P+P A-P B R^{-1} B^{T} P+Q=0$

- Construa o controle de feedback linear via:

$u=-R^{-1} B^{T} P x$

(4)

A lei de controle (3) é calculada para que o índice de desempenho é dada por $J=\int_{t_{o}}^{\infty}\left(x^{T} Q x+u^{T} R u\right) d t$ é minimizada.

A técnica do controle LQR requere que o sistema em questão seja controlável, detalhes desta técnica é mostrada nas referências [10] e [11].
A saída do sistema e os coeficientes escolhidos para as matrizes $B, Q$ e $R$ são:

$$
\begin{gathered}
B=\left[\begin{array}{llll}
1 & 0 & 0 & 0
\end{array}\right] \\
y=\left[\begin{array}{llll}
1 & 0 & 0 & 0 \\
0 & 1 & 0 & 0 \\
0 & 0 & 1 & 0 \\
0 & 0 & 0 & 1
\end{array}\right] \\
Q=\left[\begin{array}{llll}
1 & 0 & 0 & 0 \\
0 & 1 & 0 & 0 \\
0 & 0 & 1 & 0 \\
0 & 0 & 0 & 1
\end{array}\right] \\
R=1
\end{gathered}
$$

\subsection{Projeto do controle com Amortecedor Magneto Reológico}

Dentre os diversos modelos existentes para simular um Amortecedor MagnetoReológico, utilizaremos o modelo de BoucWen Modificado, presente em Dyke et al (1996), pois tem uma melhor precisão na simulação quando o sistema estudado tem alterações de velocidades muito rápidas, como o caso da suspensão automotiva.

Figura 2. Modelo físico de um Amortecedor Magneto-Reológico.

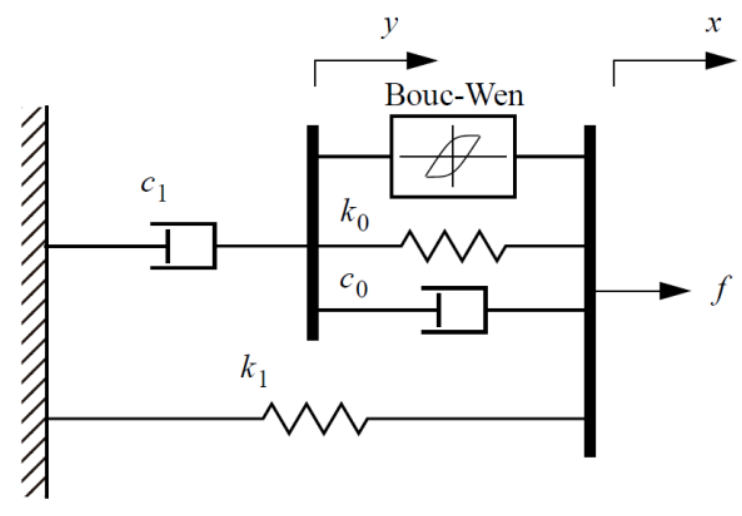

Fonte: (Dyke et al., 1996).

Em que a força desempenhada pelo amortecedor é dada por:

$f=c_{1} \dot{y}+k_{1}\left(x-x_{0}\right)$

Onde:

$$
\begin{gathered}
\dot{z}=-\gamma|\dot{x}-\dot{y}| z|z|^{n-1}-\beta(\dot{x}-\dot{y})|z|^{n} \\
+A(\dot{x}-\dot{y})
\end{gathered}
$$

Aqui, a rigidez do acumulador é representada por $k_{1}$, o amortecimento viscoso é observado em velocidades maiores 
em $c_{0}$. Um traço, representado por $c_{1}$, é incluído no modelo para introduzir o roll-off não linear nos loops força-velocidade que foi observado no dados experimentais em baixas velocidades, $k_{0}$ está presente para controlar a rigidez em grandes velocidades, e $x_{0}$ é o deslocamento inicial da mola $k_{1}$ associada à força de amortecimento nominal devido ao acumulador. Ao ajustar os parâmetros dos modelos $\gamma, \beta$ e $A$, pode-se controlar a forma dos laços de histerese para o elemento de produção.

Há também os parâmetros que relacionam a força do amortecedor com a voltagem aplicada no mesmo:

$\alpha=\alpha(u)=\alpha_{a}+\alpha_{b} u$

$c_{1}=c_{1}(u)=c_{1_{a}}+c_{1_{b}} u$

$c_{0}=c_{0}(u)=c_{0}+c_{0_{b}} u$

Sendo $u$ é dado como a saída de um filtro de primeira ordem dada por:

$\dot{u}=-\eta(u-v)$

E $v$ é o comando de tensão enviado ao controlador.

\subsection{Projeto do Controlador Híbrido}

O projeto de controle híbrido se detém na junção dos controles anteriores para que se obtenha um controle mais efetivo do sistema desejado.

Como há uma mudança no número de equações, as matrizes $y, Q$ e o coeficiente $R$ utilizados para a simulação foram:

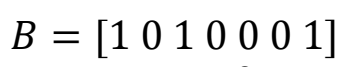

$y=\left[\begin{array}{llllll}1 & 0 & 0 & 0 & 0 & 0 \\ 0 & 1 & 0 & 0 & 0 & 0 \\ 0 & 0 & 1 & 0 & 0 & 0 \\ 0 & 0 & 0 & 1 & 0 & 0 \\ 0 & 0 & 0 & 0 & 1 & 0 \\ 0 & 0 & 0 & 0 & 0 & 1\end{array}\right]$

$Q=\left[\begin{array}{cccccc}2100 & 0 & 0 & 0 & 0 & 0 \\ 0 & 1 & 0 & 0 & 0 & 0 \\ 0 & 0 & 2100 & 0 & 0 & 0 \\ 0 & 0 & 0 & 1 & 0 & 0 \\ 0 & 0 & 0 & 0 & 1 & 0 \\ 0 & 0 & 0 & 0 & 0 & 1\end{array}\right]$

$R=1$

Para realizar as aproximações numéricas do modelo de equações, utilizouse o método de Runge Kutta de 4a Ordem implementado no software livre OCTAVE.

\section{RESULTADOS E DISCUSSÃO}

Nesta seção serão apresentados os resultados obtidos com cada controle e a análise da efetividade e robustez de cada um dos métodos.

Para efetuar a análise de dissipação da vibração, fez-se com que a pista de percurso do veículo formasse um degrau de 10 centímetros e observou-se a resposta tanto do pneu quanto da oscilação resultante ao veículo.

Os parâmetros utilizados nas simulações estão expressos na tabela 1.

As condições iniciais utilizadas para as variáveis foram as mostradas na tabela 2 . 
Tabela 1. Parâmetros utilizados nas simulações

\begin{tabular}{|c|c|}
\hline$m_{1}$ & $372 \mathrm{~kg}$ \\
\hline$m_{2}$ & $45 \mathrm{~kg}$ \\
\hline$k_{1}$ & $40000 \mathrm{~N} / \mathrm{m}$ \\
\hline$k_{2}$ & $190000 \mathrm{~N} / \mathrm{m}$ \\
\hline$c_{0_{a}}$ & $2100 \mathrm{~N} \cdot \mathrm{sec} / \mathrm{m}$ \\
\hline$c_{0_{b}}$ & $350 \mathrm{~N} \cdot \mathrm{sec} / \mathrm{m}$ \\
\hline$k_{0}$ & $4960 \mathrm{~N} / \mathrm{m}$ \\
\hline$c_{1_{a}}$ & $28300 \mathrm{~N} \cdot \mathrm{sec} / \mathrm{m}$ \\
\hline$c_{1_{b}}$ & $295 \mathrm{~N} \cdot \mathrm{sec} / \mathrm{m} \cdot \mathrm{V}$ \\
\hline$k_{1}$ & $500 \mathrm{~N} / \mathrm{m}$ \\
\hline$\alpha_{a}$ & $14000 \mathrm{~N} / \mathrm{m}$ \\
\hline$\alpha_{b}$ & $69500 \mathrm{~N} / \mathrm{cm} \cdot \mathrm{V}$ \\
\hline$\gamma$ & $3630000 \mathrm{~m}$ \\
\hline$\beta$ & $3630000 \mathrm{~m}^{-2}$ \\
\hline$A$ & 301 \\
\hline$n$ & 2 \\
\hline$\eta$ & $190 \mathrm{sec}^{-1}$ \\
\hline$v$ & $15 \mathrm{~V}$ \\
\hline
\end{tabular}

Fonte: (Kurth, 1980).

Tabela 2. Parâmetros utilizados nas simulações

\begin{tabular}{|c|c|}
\hline$x_{1}(0)$ & 0 \\
\hline$\dot{x}_{1}(0)$ & 0 \\
\hline$x_{2}(0)$ & 0 \\
\hline$\dot{x}_{2}(0)$ & 0 \\
\hline$w(0)$ & $0,1 m$ \\
\hline$\dot{w}(0)$ & 0 \\
\hline$z(0)$ & 0 \\
\hline$y(0)$ & 0 \\
\hline$u(0)$ & 0 \\
\hline
\end{tabular}

Fonte: (Roéfero e Chavarette, 2018).

\subsection{Controle LQR}

Após as simulações do sistema submetido ao controle LQR foram obtidos os seguintes resultados mostrado nas Figuras de 3 à 6. 
Figura 3. Deslocamento do pneu.

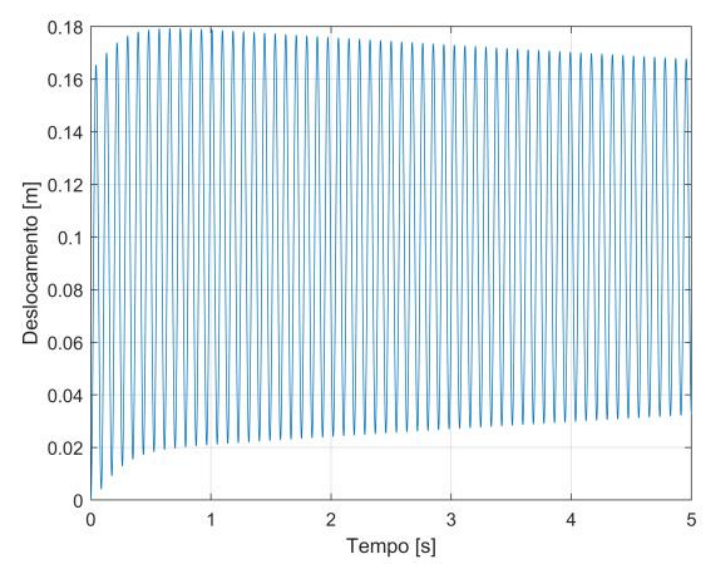

Fonte: Os autores. .

É possível perceber na Figura 3 que o controlador não controlou de forma efetiva o deslocamento do pneu do veículo, uma vez que no tempo de simulação há uma leve atenuação da vibração do sistema,

Figura 4. Deslocamento do Veículo.

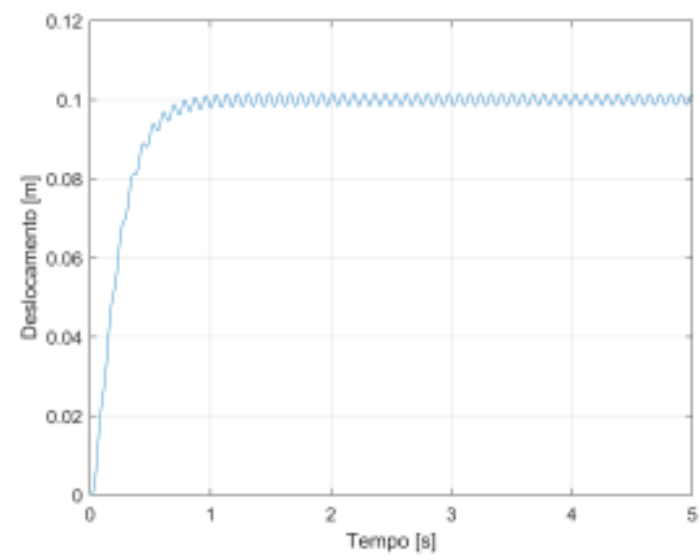

Fonte: Os autores.

Com base na Figura 4 nota-se que a carroceria do veículo foi conduzida ao ponto de equilíbrio dentro de 1 segundo após a excitação inicial. Assim, o controlador foi efetivo em seu objetivo, entretanto é possível observar uma pequena oscilação em entretanto não levando a um controle efetivo da massa $m_{2}$. 
Figura 5. Plano de fase do Pneu.

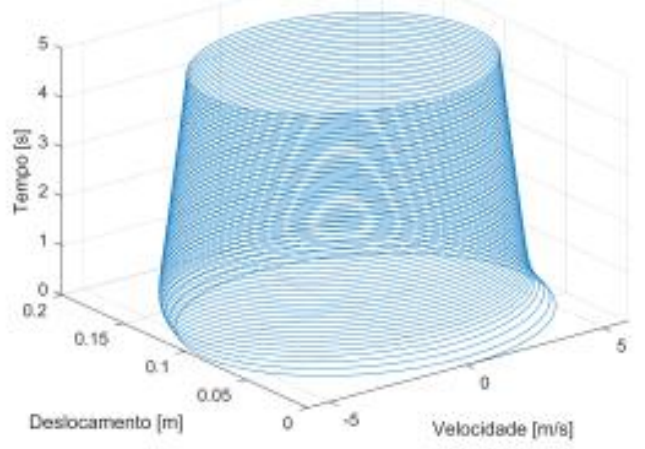

Fonte: Próprio autor.

Na Figura 5 observa-se que a órbita de oscilação do pneu no plano de fase diminui, entretanto não de forma satisfatória para

Figura 5. Plano de fase do Veículo. afirmar a robustez do controle no sistema desejado.

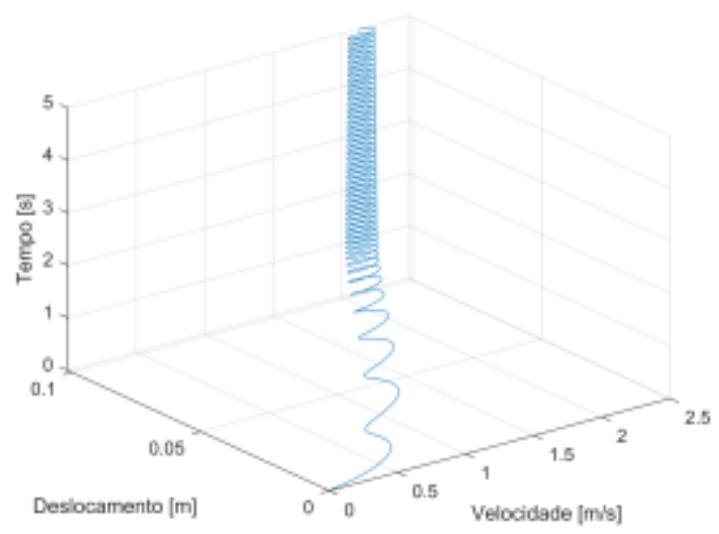

Fonte: Os autores.

Observando o plano de fase na Figura 5 relativo à massa $m_{1}$, pode-se perceber um período transiente, seguido de uma convergência para uma órbita de baixa amplitude, entretanto ainda não satisfatória para o controle desejado.

\subsection{Amortecedor Magneto Reológico} Nesta seção serão mostrados os resultados obtidos com 0 amortecedor Magneto-Reológico. 
Figura 6. Deslocamento do pneu.

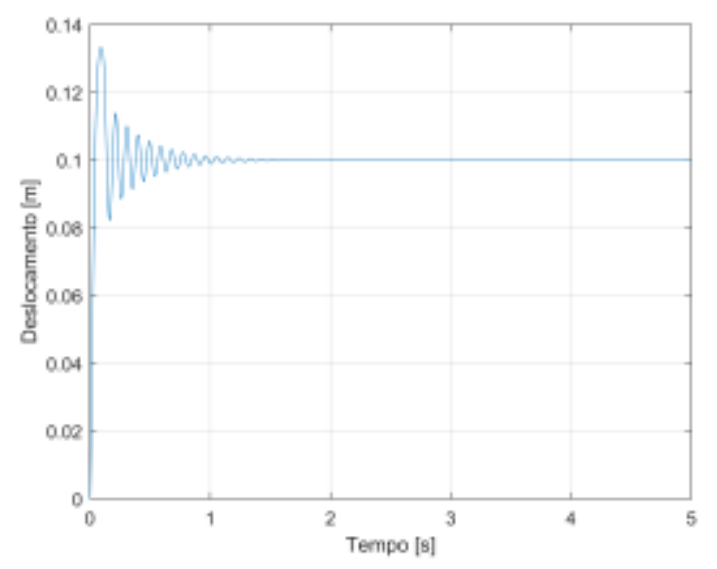

Fonte: Os autores.

Com base na Figura 6, é possível perceber que $o$ amortecedor consegue atenuar a vibração do pneu em pouco mais de 1 segundo, realizando um controle efetivo, mas não satisfatório, no sistema. Neste período de 1 segundo o motorista tem a possibilidade de perder o controle da direção do automóvel, uma vez que o pneu não se encontra em contato com o solo, levando a um possível acidente.

Figura 7. Deslocamento do Veículo.

Fonte: Os autores.

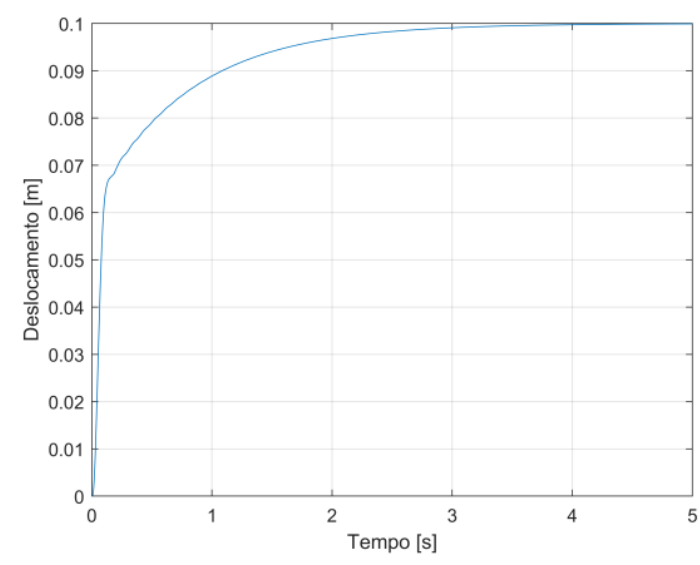

Observando a Figura 7, pode-se perceber que o veículo só converge ao ponto de equilíbrio após 4 segundos, concluindo que, embora o amortecedor seja robusto em seu controle e não permita oscilações após a estabilização, ao contrário do controlador LQR, sua resposta à excitação é demorada para a aplicação proposta.

No plano de fase mostrado na Figura 8 é possível perceber com maior clareza a convergência da órbita para o ponto de equilíbrio, como já foi discutido anteriormente. 
Figura 8. Plano de fase do pneu.

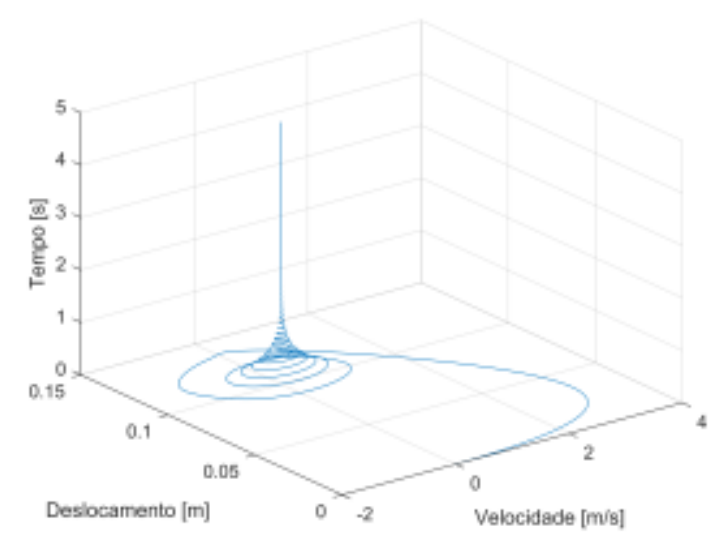

Fonte: Os autores. .

Figura 9. Plano de fase do Veículo.

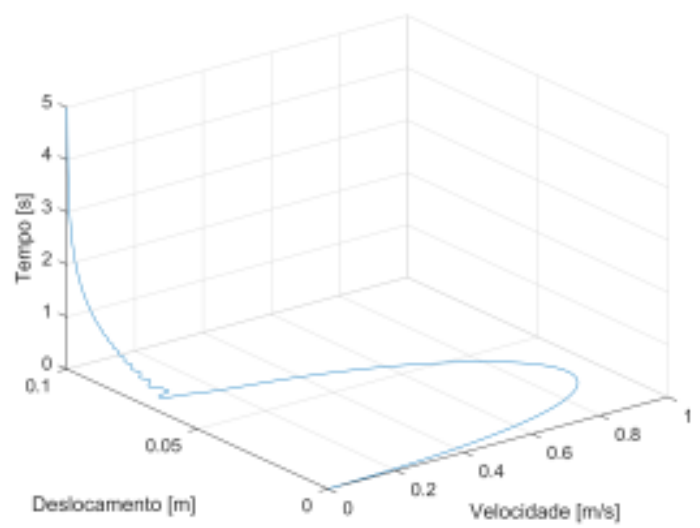

Fonte: Os autores.

No gráfico da Figura 9, é possível perceber $\mathrm{o}$ período transiente $\mathrm{e} a$ convergência ao ponto de equilíbrio que a órbita do veículo realiza, evidenciando a estabilização do mesmo.

\subsection{Controlador Híbrido}

Com base nos resultados obtidos anteriormente com os controles aplicados isoladamente, decidiu-se projetar um controle híbrido a fim de obter um melhor resultado.

Com base na Figura 7, é possível perceber que o controlador híbrido consegue atenuar a vibração do pneu em aproximadamente 0,5 segundos, resultando em um amortecimento gradual que permite que o motorista do veículo tenho o controle do veículo, tornando 0 resultado bastante satisfatório.

Observando a Figura 11, podemos perceber que 0 veículo converge ao ponto de equilíbrio em menos de um segundo e sem deixar qualquer órbita de oscilação, tornando o controlador híbrido efetivo em seu projeto de controle.

No plano de fase mostrado na Figura 12 é possível perceber com maior clareza a convergência da órbita para o ponto de equilíbrio de forma rápida e efetiva. 
Figura 10. Deslocamento do pneu.

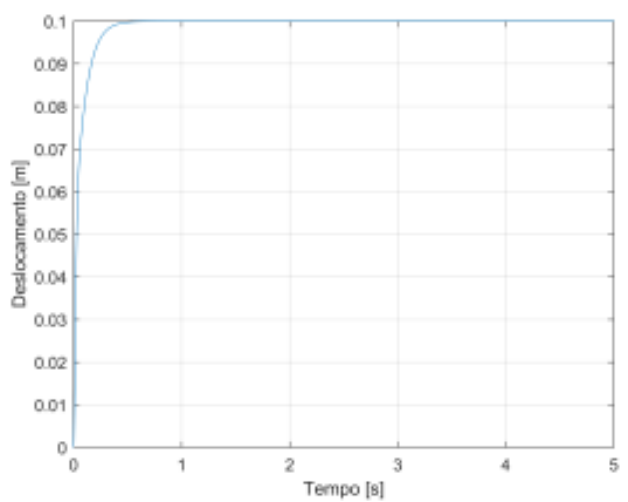

Fonte: Os autores. .

Figura 11. Deslocamento do Veículo.

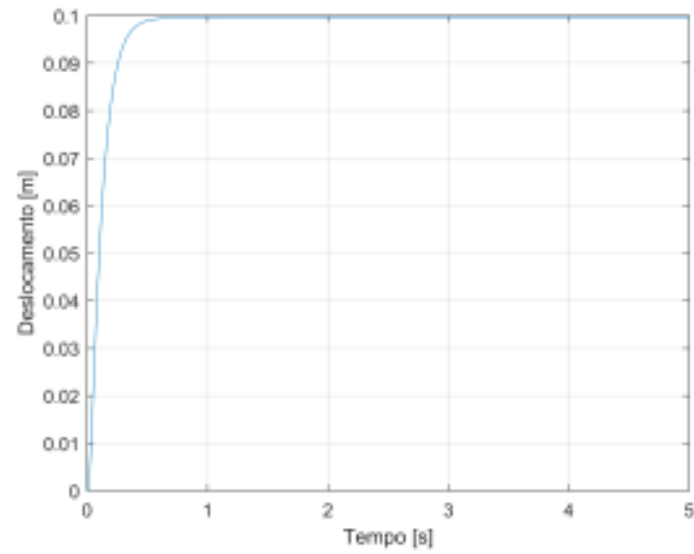

Fonte: Os autores.

Figura 12. Plano de fase do pneu.

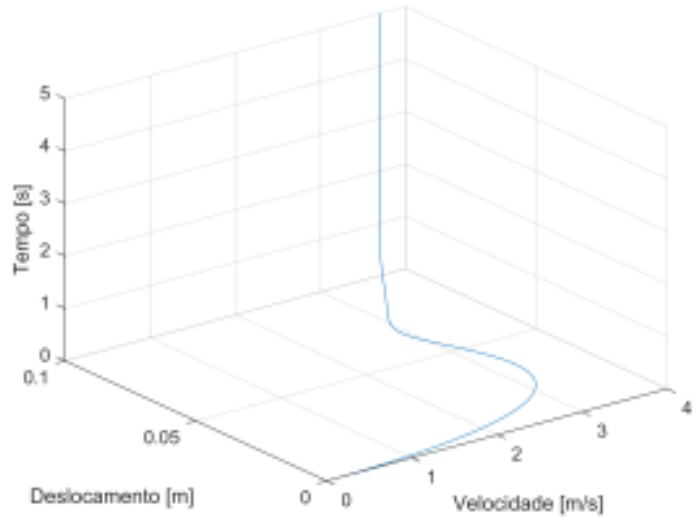

Fonte: Os autores. 
Figura 13. Plano de fase do Veículo.

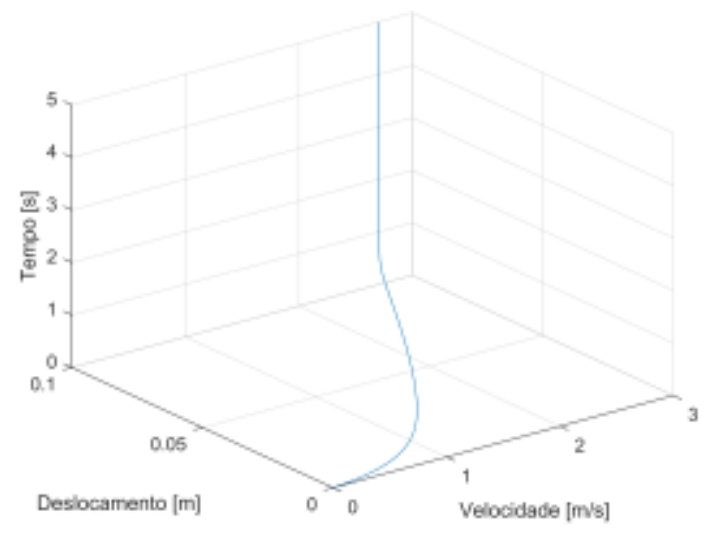

Fonte: Os autores.

No gráfico da Figura 13, é possível perceber o período transiente e a convergência ao ponto de equilíbrio que a órbita do veículo realiza, evidenciando a estabilização do mesmo e sem qualquer oscilação sobre o ponto de equilíbrio.

\section{CONSIDERAÇÕES FINAIS}

Com base na teoria enunciada neste trabalho, no procedimento empregado para a elaboração dos algoritmos, os quais visavam o cumprimento de um objetivo proposto, que era a análise de métodos de controle empregados na atenuação de vibração em uma suspensão automotiva. É possível perceber que dentre os três métodos de controle empregados, o controle híbrido correspondeu melhor aos resultados de suavização de vibração no automóvel.

Este melhor desempenho se deve ao fato de o controle híbrido compreender a rapidez de convergência ao ponto de equilíbrio promovida pelo Controlador Linear Quadrático (LQR), bem como a supressão de pequenas vibrações promovida pelo amortecedor magneto-reológico. Assim, percebe-se que o controle é mais efetivo quando há a junção dos dois controladores, formando o controle híbrido em questão.

AGRADECIMENTOS
Os autores agradecem ao laboratório SISPLEXOS e a UNESP por oferecer sua estrutura como suporte acadêmico à pesquisa, bem como à Fundação de Amparo à Pesquisa do Estado de São Paulo (FAPESP), processo № 2017/17601-8 pelo apoio financeiro ao trabalho.

\section{REFERÊNCIAS}

ALVAREZ-SÁNCHEZ, E. A Quarter-car Suspension System: Car Body Mass Estimator and Sliding Mode Control In: IBEROAMERICAN CONFERENCE ON ELECTRONICS ENGINEERING AND COMPUTER SCIENCE, 1., 2013, Rio de Janeiro. Anais [...] Xalapa: Elsevier, 2013. p. 208-214.

BUENO, D. D. Controle ativo de vibrações e localização ótima de sensores e atuadores piezelétricos. 2007. Dissertação (Mestrado) Faculdade de Engenharia, Universidade Estadual Paulista, Ilha Solteira. 2007.

CABRAL, T. D. F.; CHAVARETTE, F. R. Dynamics and control design via LQR and SDRE methods for a maglev system. International Journal of Pure and Applied Mathematics, v. 101, n. 2, p. 289300, 2015.

CUNHA, B. S. C.; CHAVARETTE, F. R. Vibration Control Applied in a Semi-Active Suspension 
Using Magneto Rheological Damper and Optimal Linear Control Design. Applied Mechanics and Materials, v. 464, p. 229-234, 2013.

https://doi.org/10.4028/www.scietific.net/A MM.464.229

DE LIMA, A. M. G. Modélisation et Optimisation Robuste de l'amortissement Viscoélastique de Systèmes Mécaniques. 2007. Tese (Doutorado) - Université de Franche-Comté, 2007.

DYKE, S. J.; SPENCER Jr, B. F.; SAIN, M. K.; CARLSON, J. D. Modeling and control of magnetorheological dampers for seismic response reduction. Smart materials and structures, v. 5, n. 5, p. 565, 1996. https://doi.org/10.1088/0964-1726/5/5/006

GNU Octave 2.4.0 Released, 2018.

PICADO, R. M. Controle Semi-Ativo em Suspensões Automotivas. 1998. Dissertação (Mestrado) - Universidade Estadual de Campinas, Campinas. 1998. Disponível em: http://repositorio.unicamp.br/bitstream/REP OSIP/264369/1/Picado_RicardoMigueis_M.p df. Acesso em: 01 ago. 2018.

ROÉFERO, L. G. P.; CHAVARETTE, F. R. Controse Semi-Ativo Aplicado a Vibrações Excessivas de uma Suspensão Automotiva. Colloquium Exactarum. v.10, n.3, p.16-22, 19 de. 2018. https://doi.org/10.5747/ce.2018.v10.n3.e242

TUSSET, A. M.; BALTHAZAR, J. M.; CHAVARETTE, F. R.; FELIX, J. L. P. On Energy transfer phenomena, in a nonlinear ideal and non-ideal essential vibrating systems, coupled to a (MR) Magneto-rheological damper. Nonlinear Dynamics, v. 69, p. 18591880, 2012.

https://doi.org/10.1007/s11071-012-0391-5 